\title{
Relación de Supervisión: Adaptación del S-SRQ en Psicoterapeutas Argentinos
}

\section{Supervision Relationship: Adaptation of the S-SRQ in Argentine Psychotherapists}

\author{
Pablo R. Santangelo 10 RCID, Karina Conde $\underline{O R C I D}$
}

Universidad Nacional de Mar del Plata.

Argentina

Fecha correspondencia:

Recibido: agosto 21 de 2019.

Aceptado: julio 17 de 2020.

Forma de citar:

Santangelo, P.R., \& Conde, K. (2020).

Relación de Supervisión: Adaptación

del S-SRQ en Psicoterapeutas

Argentinos. Rev. CES Psico, 13(3),

76-87.

\section{Open access}

(c) Copyright

Licencia creative commons

Ética de publicaciones

Revisión por pares

Gestión por Open Journal System DOI: http://dx.doi.org/10.21615/

cesp.13.3.5

ISSN: 2011-3080

Sobre los autores:

1. Especialista en Psicoterapia Individual y Grupal. Director del Programa de Formación y Entrenamiento en Psicoterapia y del Proyecto de Investigación "Psicoterapia y Supervisión Clínica: Habilidades, Competencias

Comparte

\section{Resumen}

Introducción: En los últimos años se ha reconocido la importancia de evaluar la supervisión clínica y su rol en el proceso psicoterapéutico. No obstante, son escasos los instrumentos que cumplen con este propósito, particularmente en Latinoamérica. En este estudio, se buscó adaptar al castellano la versión breve del Cuestionario de relación de supervisión (S-SRQ, por sus siglas en inglés de: Short Supervisory Relationship Questionnaire), compuesto de tres dimensiones: base segura, educación reflexiva y estructura; y aportar evidencias sobre su desempeño psicométrico. Método: El S-SRQ fue traducido y retraducido a su idioma original. Posteriormente, fue administrado a 374 psicoterapeutas argentinos (muestra no probabilística). Se realizaron análisis de consistencia interna (alfa de Cronbach), dimensionales (análisis paralelo y factorial confirmatorio), y a nivel del ítem (coeficientes estandarizados y correlación ítem-escala). Resultados: Los coeficientes de consistencia interna fueron adecuados para la escala total y, en general, para cada dimensión. Los análisis dimensionales se ajustaron a un modelo de tres dimensiones, base segura, educación reflexiva y estructura. El desempeño de los ítems fue bueno con relación a la escala total y cada dimensión. Discusión: El desempeño psicométrico del S-SRQ en castellano fue similar al de la escala original, con algunas diferencias. La escala presenta potencial para ser aplicada en el contexto argentino, tanto en el ámbito científico como en la práctica.

Palabras claves: Relación de Supervisión, Evaluación de Supervisión, Psicoterapia, Adaptación, Supervisión Clínica, Psicología Clínica, Supervisión en Psicología, Supervisión en Psicoterapia, Investigación en Psicoterapia.

\section{Abstract}

Introduction: In recent years the significance of evaluating clinical supervision and its role in the psychotherapeutic process has been recognized. However, there are few instruments for such evaluation, particularly in Latin America. This study aimed to adapt the three-dimensional (reliable basis, reflexive education and structure) Short Version of the Supervisory Relationship Questionnaire (S-SRQ) to Spanish. Method: The questionnaire was administered to 374 Argentine psychotherapists (non-probabilistic sample). The S-SRQ was translated and retranslated to its original language. It was performed internal 
Terapéuticas y Resultados de Tratamientos", Coordinador docente de la Carrera de Especialización en Psicoterapia Cognitiva (Fundación Aiglé), Docente de Grado y Postgrado, Universidad Nacional de Mar del Plata. Coordinador de CAPsi, Centro de Asistencia Psicológica, Mar del Plata.

2. Dra. en Psicología. Investigadora Asistente del Instituto de Psicología Básica, Aplicada y Tecnología (IPSIBAT), Consejo Nacional de Investigaciones Científicas y Técnicas (CONICET). Universidad Nacional de Mar del Plata. consistency (Cronbach's alpha), dimensional (parallel and confirmatory factor analysis), and item analyzes (standardized coefficients and item-scale correlation). Results: The internal consistency coefficients were adequate for the total scale and, in general, for each dimension. The dimensional analyses were adjusted to a three-dimensional model, reliable basis, reflective education and structure. Item performance was good in relation to the total scale and each dimension. Discussion: The psychometric performance of the S-SRQ in Spanish was like the original scale, with some exceptions. The scale has potential to be implemented in our context, both at a practical and scientific level.

Keywords: Supervision Relationship, Supervision Measures, Psychotherapy, Adaptation, Clinical Supervision, Clinical Psychology, Psychology Supervision, Psychotherapy Supervision, Psychotherapy Research.

\section{Introducción}

La supervisión en psicoterapia es una intervención provista por un miembro con más experiencia y pericia en el ejercicio de la psicoterapia (supervisor) a otros colegas con menos experiencia (supervisados), con el propósito simultáneo de facilitar y mejorar el desempeño profesional de los supervisados y monitorear la calidad de los servicios profesionales ofrecidos a los pacientes, y de este modo, proteger su bienestar (Bernard \& Goodyear, 2019; Falender \& Shafranske, 2004). La supervisión debe estar enfocada en las necesidades de los supervisados y las tareas claves del proceso; y el contrato realizado entre supervisor y supervisado y la retroalimentación, son elementos que permitirán el desarrollo de la relación de supervisión (Beinart \& Clohessy 2017).

Durante mucho tiempo, la práctica de la supervisión en psicoterapia ha sido un factor importante en el proceso de capacitación de los psicoterapeutas y el mecanismo más relevante para desarrollar sus competencias durante el entrenamiento (Falender et al., 2004; Stoltenberg, 2005; Bernard \& Goodyear, 2014). Se ha demostrado su impacto beneficioso para el desempeño de los supervisados en aspectos como mayor autoconciencia, conocimiento, adquisición y uso de habilidades, autoeficacia, fortalecimiento de la relación entre supervisado y paciente, y disminución de la ansiedad del profesional (Inman et al. 2014: Gibson, Gray, \& Hastings, 2009; Goodyear \& Guzzardo, 2000; Holloway, 2012; Inman \& Ladany, 2008; Ladany \& Inman, 2011; Wheeler \& Richards, 2007). Sin embargo, también se han reportado consecuencias adversas que afectarían la relación entre supervisado y supervisor, por ejemplo, el supervisado al sentir que su supervisor lo juzga no revela la totalidad de la información respecto a las intervenciones que ha realizado con el paciente (Gray, Ladany, Walker, \& Ancis, 2001; Mehr, Ladany, \& Caskie, 2010). En este sentido se ha identificado que una práctica inadecuada de supervisión puede generar malestar en el supervisado, como reacciones de ansiedad y preocupación (Ellis et al., 2013). En los pacientes, el impacto de la supervisión ha sido más difícil de medir y ha permanecido en gran parte como un territorio inexplorado (Hill \& Knox, 2013), con resultados contradictorios (Freitas, 2002; Milne, Sheikh, Pattison, \& Wilkinson, 2011; Watkins, 2011).

Lo cierto es que gran parte de la investigación en el campo de la supervisión en psicoterapia se ha desarrollado en torno a su conceptualización y evaluación (Bernard \& Goodyear, 2019; Cliffe, Beinart, \& Cooper, 2016). La importancia de la relación de supervisión (RS) ha sido señalada por diversos autores (Ellis, 2010; Watkins, 2014); e identificada, recientemente, por Watkins (2018) como un elemento clave de la práctica de la supervisión en el ámbito de la psicoterapia. La RS se caracteriza por la colaboración y el trabajo mutuo, está mediada por el respeto, y es abierta, en el sentido que se basa en la retroalimentación brindada por el supervisado (Beinart \& Clohessy 2017). Una de las primeras autoras en estudiar el concepto de RS fue Holloway (1995), quien lo definió como un proceso 
Pág 78

A partir de una revisión conceptual de la relación de supervisión (RS) y psicométrica de escalas que la evalúan, Tangen y Borders (2016) recomiendan el S-SRQ para evaluar las tres dimensiones que facilitarían una supervisión efectiva. dinámico en el cual el supervisor y el supervisado negocian una manera personal de utilizar una estructura de poder y participación que se acomoda a la progresión del aprendizaje del supervisado, durante las distintas fases del proceso.

Según Beinart (citado por Reiser \& Watkins, 2014) la evaluación de la RS mediante métodos cuali-cuantitativos sugirió que la satisfacción con la supervisión, la relación (o vínculo) entre el supervisor y el supervisado, y sentirse apoyado por el supervisor son las principales cualidades que los supervisados consideran favorecedoras de su efectividad como profesionales. Además, se identificaron nueve categorías que describen la calidad de la RS: 1) encuadre, 2) apoyo, 3) relación abierta, 4) respeto, 5) compromiso, 6) sensibilidad a las necesidades, 7) colaboración, 8) educación y 9) evaluación. Este modelo se utilizó posteriormente para desarrollar el Cuestionario de Relación de Supervisión, SRQ (por sus siglas en inglés de: Short Supervisory Relationship Questionnaire) una medida psicométricamente sólida de la RS desde la perspectiva de los supervisados (Palomo, Beinart, \& Cooper, 2010). Esta versión de 67 ítems fue reducida a una versión de 18 ítems y tres dimensiones, dando lugar al S-SRQ, el cual presentó propiedades psicométricas satisfactorias (Cliffe et al., 2016). Otros estudios coinciden en señalar la relevancia de estas tres dimensiones en la RS (Gibson, Ellis, \& Friedlander, 2019; Kennedy, Keaney, Shaldon, \& Canagaratnam, 2018; Watkins \& Riggs, 2012), a saber: a) Base segura, incluye las características básicas de una relación de supervisión como la colaboración, el respeto, no juzgar y sentirse seguro; las cuales le facilitan al supervisado discutir abiertamente sus preocupaciones. Y dado que se trata de experimentar y hablar sobre el contenido emocional del trabajo clínico, la contención emocional es un factor necesario en la RS (Milne et al., 2011). La dimensión Educación Reflexiva se refiere a los procesos de reflexión y aprendizaje que son importantes para facilitar una supervisión efectiva. Este componente incluye elementos sobre la capacidad del supervisor para utilizar una gama de modelos teóricos y alentar a sus supervisados a reflexionar sobre sus prácticas y necesidades de aprendizaje; para prestar atención al proceso de supervisión, y para supervisar los sentimientos y ansiedades no expresados. Hay consenso sobre la función formativa de la supervisión y la importancia de facilitar el aprendizaje como principios de la supervisión basada en la evidencia (Beinart, 2012; Bernard \& Goodyear, 2014; Milne, 2017). Y la dimensión Estructura se relaciona con los aspectos organizativos de la supervisión, por ejemplo, si las sesiones están lo suficientemente enfocadas, estructuradas y continuas, los límites y la conformación del proceso.

A partir de una revisión conceptual de la RS y psicométrica de escalas que la evalúan, Tangen y Borders (2016) recomiendan el S-SRQ para evaluar las tres dimensiones que facilitarían una supervisión efectiva. En Argentina no se ha validado ningún instrumento que evalúe la RS, si bien algunos autores están trabajando en la adaptación de una escala que evalúa el constructo (Fernández Álvarez \& Maristany, comunicación personal, junio de 2018). Así, el presente estudio tiene como objetivo adaptar al castellano la versión breve del S-SRQ (Cliffe et al., 2016), y de aportar evidencias sobre su desempeño psicométrico.

\section{Método}

Es un estudio no experimental, correlacional con el objetivo de adaptar el S-SRQ al contexto argentino.

\section{Participantes}

Se estimó un tamaño de muestra >200 personas, necesario para la realización de análisis factoriales confirmatorios con variables ordinales (Flora \& Curran, 2004). La muestra estuvo constituida por 374 psicólogos que ejercían como psicoterapeutas y concurrían a sesiones de supervisión. El $88 \%$ de sexo femenino, con una media de edad de $M=35.95(D E=8.41)$, una $M=5.98(D E=5.76)$ de años en ejercicio de la psicoterapia y una 
Pág 79

Se estimó un tamaño de muestra $>200$ personas, necesario para la realización de análisis factoriales confirmatorios con variables ordinales (Flora \& Curran, 2004). La muestra estuvo constituida por 374 psicólogos que ejercían como psicoterapeutas y concurrían a sesiones de supervisión.
$M=3.79(D E=2.81)$ de horas de supervisión semanales. En su mayoría trabajaban desde un modelo teórico integrativo (45\%), seguido de los modelos psicoanalítico $(27 \%)$ y cognitivo (24\%), y en menor medida humanístico (3\%) y sistémico (1\%).

\section{Instrumento}

La versión corta del Cuestionario de Relación de Supervisión -S-SRQ- (por sus siglas en inglés Short Supervisory Relationship Questionnaire) (Cliffe et al., 2016) cuenta 18 ítems de la versión original y evalúa tres dimensiones de la RS, a saber, Base Segura, Educación Reflexiva y Estructura. Las opciones de respuesta conforman un continuo que va de fuertemente en desacuerdo (1) a fuertemente de acuerdo (7); y el ítem 17 tiene puntuación inversa.

Fue validado con una muestra de 220 psicoterapeutas, siendo el componente Base Segura el que representó la mayor proporción de varianza (57\%), Educación Reflexiva representó el $7 \%$ y Estructura el $5 \%$. La consistencia interna de la escala general fue alta $(\alpha=.96)$ y las correlaciones ítems-totales variaron de .53 a .87. Los valores de consistencia interna para las subescalas (y los rangos para correlaciones ítem-total) fueron los siguientes: Base Segura $\alpha=.97$ (rango .79 a 90), Educación Reflexiva $\alpha=.89$ (rango .67 a .80), Estructura $\alpha=.88$ (rango .69 a .78 ).

La fiabilidad test-retest fue aceptable, y la validez convergente fue probada con correlaciones positivas con otras medidas de relación de supervisión incluida el SQR, y correlaciones negativas con una medida del conflicto de roles de supervisión y la ambigüedad del rol (Olk \& Friedlander, 1992). La validez divergente demostró ser buena al no presentar relación significativa con los puntajes del Cuestionario breve de personalidad de Eysenck revisado (Eysenck, Eysenck, \& Barrett 1985). El S-SRQ tuvo una buena validez predictiva, a partir de análisis de regresión lineal y múltiple, en relación con las calificaciones de eficacia y satisfacción de la supervisión (Cliffe et al., 2016).

\section{Procedimiento}

El S-SRQ fue adaptado siguiendo las Normas de la Comisión Internacional de Tests (Asociación Argentina de Estudio e Investigación en Psicodiagnóstico, 2000) y el método de traducción inversa, a fin de generar una versión que se corresponda con el idioma local y los estilos lingüísticos propios del contexto en el que se aplicó. Cabe destacar que en la traducción participó la Dra. Helen Beinart, una de las autoras del instrumento. La escala traducida fue sometida a examen y juicio crítico de un grupo de investigación conformado por psicólogos y un especialista en la construcción de instrumentos de evaluación psicológica; quienes estuvieron de acuerdo con la traducción, que no presentó modificaciones respecto a la escala original. Por último, con el fin de evaluar el nivel de comprensión de los ítems, se realizó una prueba piloto con 15 psicólogos, que se encontraban en procesos de supervisión, con más de cinco años de experiencia; y durante la prueba se dio la posibilidad de realizar comentarios respecto de la versión traducida del S-SRQ y ninguno tuvo dudas ni sugerencias respecto a los ítems.

Posteriormente, se administró esta versión de manera individual, presencial y on-line, a la muestra conformada por 374 de psicólogos. Los participantes contestaron los cuestionarios de manera presencial, 27\% y online, 73\%. La recolección de datos se realizó entre marzo del año 2018 y febrero de 2019. 


\section{Consideraciones éticas}

Todos participaron voluntariamente y no recibieron ningún tipo de compensación; además, recibieron información general sobre el estudio y firmaron el consentimiento informado, asegurando el anonimato y confidencialidad de la información otorgada, y se incluyeron datos de contacto de los responsables del estudio. Este estudio se adhiere a los principios éticos enmarcados en la Declaración de Helsinki.

\section{Análisis de datos}

La consistencia interna de la escala total y las subescalas se estableció mediante el coeficiente Alfa de Cronbach y el Lambda 6 de Guttman ( $\lambda 6$ ). La valoración de la dimensionalidad de la escala se realizó mediante análisis paralelo (número de replicaciones $=100$, percentil de representación de simulaciones $=.95$ ) y análisis factoriales confirmatorios (AFC), considerando las dimensiones teóricas originales de la escala como factores no relacionados. Dado que los ítems poseen una distribución no paramétrica, se utilizó el estimador Diagonally Weighted Least Squares (DWLS) (Brown, 2006). El ajuste a este modelo (Figura 1) se valoró con los índices X2, Comparative Fit Index (CFI, valor de referencia $\geq .90$ ), Tucker-Lewis Index (TLI, valor de referencia $=\geq$.90), Root Mean Square Error of Approximation (RMSEA, valor de referencia $\leq .05)$, y Standardized Root Mean Square Residual (SRMR, valor de referencia $\leq .05)$ (Hu \& Bentler, 1999). El punto de corte para considerar las cargas factoriales como apropiadas fue de $\lambda<.30$ (Kline, 2013). Para la evaluación individual de los ítems se tuvieron en cuenta los coeficientes estandarizados y la correlación puntual biserial de cada uno con la subescala. Para los análisis se utilizaron los paquetes estadísticos Psych, (Revelle, 2012), y Lavaan (Rosseel, 2012) del software R 3.5.3.
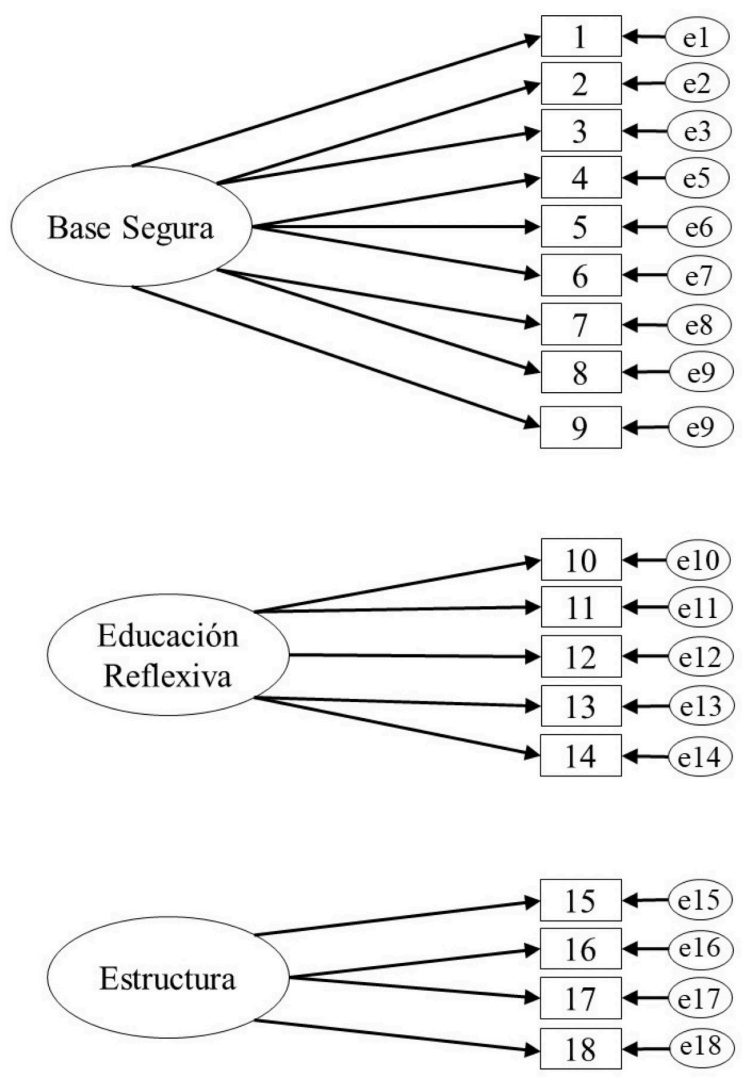

Figura 1. Modelo evaluado mediante Análisis Factorial Confirmatorio para el Short Version of the Supervisory Relationship Questionnaire - S-SRQ. 


\section{Resultados}

En primer lugar, se evaluó la fiabilidad de la escala total y de cada dimensión. Los coeficientes de confiabilidad fueron adecuados para la escala total $(\alpha=.86, \mathrm{IC} 95 \%=.84-88, \lambda 6=.9$ ). Para cada una de las dimensiones la confiabilidad en general fue aceptable: Base Segura $(\alpha=.86$, IC95\% $=.84-.88, \lambda 6=.86)$, Educación Reflexiva ( $\alpha=.72$, IC95\%=.68-.76, $\lambda 6=.73)$, Estructura $(\alpha=.56, \mathrm{IC} 95 \%=.5-.62, \lambda 6=.51)$.

Se prosiguió entonces con el análisis dimensional de la escala. El análisis paralelo sugirió la presencia de tres factores por análisis factorial, y dos por análisis de componentes principales. A partir del AFC se detectó que el ajuste del modelo de tres dimensiones fue satisfactorio $X^{2}=115.18, \mathrm{gl}=132, p=.851, \mathrm{CFI}=1.00, \mathrm{TLI}=1.01, \mathrm{RMSEA}=0.01, \mathrm{SRMR}=0.06$, con coeficientes estandarizados por encima de .40 para todos los ítems (Figura 2). Las covarianzas entre las dimensiones fueron: Base Segura y Educación Reflexiva, $r=.87, p=.001$; Base Segura y Estructura, $r=.56, p=.001$; Educación Reflexiva y Estructura, $r=.65, p=.001$.
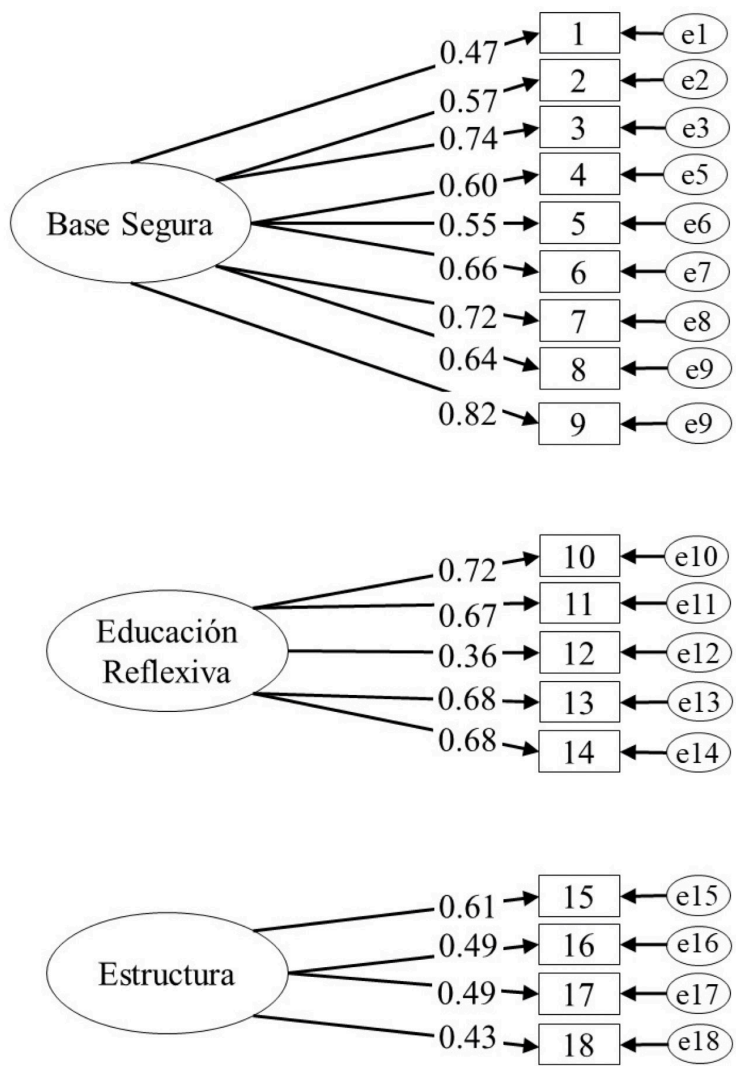

Figura 2. Coeficientes estandarizados para el Análisis Factorial Confirmatorio de la Escala (Short Version of the Supervisory Relationship Questionnaire - S-SRQ) Psicoterapeutas argentinos, $n=374$

Por último, se realizó un análisis a nivel del ítem. Todos los ítems presentaron en todos los casos correlaciones superiores a .30, con excepción del ítem 16 y el puntaje total (Tabla 1). 
Tabla 1. Análisis a nivel del ítem (correlación biserial puntual corregida) de la Escala

\begin{tabular}{|c|c|c|}
\hline Ítems & Total* $^{*}$ & Dimensión ${ }^{*}$ \\
\hline \multicolumn{3}{|l|}{ Base Segura } \\
\hline 1. Mi supervisor estuvo disponible & .52 & .49 \\
\hline 2. Mi supervisor respetó mis opiniones e ideas & .55 & .61 \\
\hline $\begin{array}{l}\text { 3. Mi supervisor me trasmitió sus comentarios de una } \\
\text { manera que me dio seguridad }\end{array}$ & .69 & .73 \\
\hline 4. Mi supervisor estuvo entusiasmado de supervisarme & .61 & .59 \\
\hline $\begin{array}{l}\text { 5. Me sentí capaz de discutir abiertamente mis } \\
\text { preocupaciones con mi supervisor }\end{array}$ & .52 & .55 \\
\hline 6. Mi supervisor no me juzgó/criticó & .60 & .66 \\
\hline 7. Mi supervisor tuvo una mente abierta en la supervisión & .69 & .71 \\
\hline $\begin{array}{l}\text { 8. Mi supervisor me realizó comentarios / devoluciones } \\
\text { positivas sobre mi desempeño }\end{array}$ & .62 & .63 \\
\hline $\begin{array}{l}\text { 9. Mi supervisor tuvo un enfoque colaborativo en la } \\
\text { supervisión }\end{array}$ & .79 & .81 \\
\hline \multicolumn{3}{|l|}{ Educación Reflexiva } \\
\hline 10. Mi supervisor me animó a reflexionar sobre mi práctica & .68 & .69 \\
\hline $\begin{array}{l}\text { 11. Mi supervisor prestó atención a mis sentimientos no } \\
\text { expresados y ansiedades }\end{array}$ & .60 & .68 \\
\hline $\begin{array}{l}\text { 12. Mi supervisor fue flexible sobre diferentes modelos } \\
\text { teóricos }\end{array}$ & .34 & .36 \\
\hline $\begin{array}{l}\text { 13. Mi supervisor puso mucha atención al proceso de } \\
\text { supervisión }\end{array}$ & .68 & .63 \\
\hline $\begin{array}{l}\text { 14. Mi supervisor me ayudó a identificar qué necesito } \\
\text { aprender y entrenar }\end{array}$ & .62 & .66 \\
\hline \multicolumn{3}{|l|}{ Estructura } \\
\hline 15. La supervisión fue focalizada & .43 & .56 \\
\hline 16. La supervisión fue estructurada & .28 & .50 \\
\hline 17. Mis sesiones de supervisión fueron desorganizadas & .35 & .45 \\
\hline $\begin{array}{l}\text { 18. Mi supervisor se aseguró que las sesiones no fueran } \\
\text { interrumpidas }\end{array}$ & .39 & .38 \\
\hline
\end{tabular}

Nota. ${ }^{*}$ Calculado en base al total de la escala ¥Calculado en base a la dimensión correspondiente

En síntesis, el S-SRQ resultó tener índices adecuados que aportan a su validez en el contexto local. 
Respecto a su aplicación practica, esta adaptación permitiría la utilización del S-SRQ en nuestro contexto (Argentina) como un instrumento para evaluar el modo como los supervisados valoran la RS, variable fundamental del proceso de supervisión en psicoterapia. También permitiría al supervisor adaptar y ajustar el proceso de supervisión a las necesidades de los supervisados.

\section{Discusión}

El objetivo del presente estudio fue adaptar al castellano la versión breve del Cuestionario de Relación de Supervisión -S-SRQ- (Cliffe et al., 2016) y proporcionar evidencias sobre su desempeño psicométrico. De acuerdo con los hallazgos, el S-SRQ presentó índices adecuados, tanto a nivel general como a nivel del ítem; por lo cual es recomendable su aplicación en nuestro contexto (Argentina).

Los coeficientes de confiabilidad de la escala total y de cada una de las subescalas encontrados en la presente investigación, fueron levemente menores que los de la escala original (Cliffe et al., 2016) y los menores puntajes se obtuvieron en las subescalas Educación Reflexiva y Estructura, tal como en el estudio de Cliffe et al. (2016). A pesar de la menor consistencia interna o valor $\alpha$, el ajuste general del modelo de tres dimensiones fue bueno ( $p>$.05, CF>90, TLI>90, RMSEA<0.05), como también el de cada ítem y la dimensión a la cual pertenecían. En concordancia con lo anterior, el desempeño de los ítems fue óptimo en casi todos los casos, dado que la correlación entre cada uno y las dimensiones a las que pertenecían fue aceptable (>30 en todos los casos), a pesar de la confiabilidad menor en algunas de ellas. El único ítem que presentó baja correlación con la escala total fue "La supervisión fue estructurada"; resultado que podría obedecer a que el término "estructurada" no es un descriptor adecuado para la práctica de la RS en nuestro contexto, o podría resultar vago para los participantes.

Las propiedades psicométricas de la escala son aceptables y las dimensiones de la misma tienen argumentos a favor de su utilidad en la supervisión clínica (Barnett, Erickson Cornish, Goodyear, \& Lichtenberg, 2007; Milne et al., 2011; Scaturo \& Watkins, 2014; Watkins, 2012).

El modelo presentado se basa en el constructo RS, creado para entender cómo funciona la supervisión clínica, compuesto por tres dimensiones: Base Segura, Educación Reflexiva y Estructura; teniendo en cuenta esta consideración, cabe una reflexión teórica en relación con otros conceptos. En la literatura sobre la supervisión los términos alianza de trabajo en supervisión (ATS) y relación de supervisión (RS) tienden a usarse indistintamente; sin embargo, la ATS es una construcción teórica de Bordin (1983), que proviene de los desarrollos de la alianza de trabajo en psicoterapia y hace referencia al acuerdo mutuo sobre los objetivos y tareas de supervisión, y el vínculo que se desarrolla entre el supervisor y supervisado. Aunque algunos autores sugieren que los supervisores deben monitorear la alianza de trabajo siguiendo la línea de Bordin y hacer explicito el trabajo en colaboración (Gibson et al., 2019), otros han argumentado que confiar en los modelos propios de la psicoterapia y extrapolarlos a la supervisión puede ser restrictivo, porque la supervisión y la psicoterapia son fundamentalmente diferentes. La supervisión clínica es principalmente formativa/educativa, implica un activo monitoreo y tiene una función de control de calidad (Beinart, 2012; Bernard \& Goodyear, 2014; Palomo et al., 2010; Simon, Cruise, Huber, Swerdlik, \& Newman, 2014; Tangen \& Borders, 2016); por lo tanto, la RS, concebida a partir de aspectos como metas, tareas y vínculo, se considera una explicación parcial de la misma. Es por esta razón que la línea de investigación que evalúa la RS en un sentido más amplio se ajusta más a lo que pasa dinámicamente dentro del espacio de supervisión.

Respecto a su aplicación práctica, esta adaptación permitiría la utilización del S-SRQ en nuestro contexto (Argentina) como un instrumento para evaluar el modo como los supervisados valoran la RS, variable fundamental del proceso de supervisión en psicoterapia. También permitiría al supervisor adaptar y ajustar el proceso de supervisión a las necesidades de los supervisados. El ajuste de la intervención a partir de las respuestas redundaría en un mejor trabajo en el espacio de supervisión, en el desarrollo del psicoterapeuta 
y posiblemente en los resultados de los tratamientos. Así mismo, puede ser utilizada, en el campo científico, para la evaluación del impacto de la RS sobre los resultados de los tratamientos (Cliffe et al., 2016) y el desarrollo de habilidades y competencias terapéuticas.

Es necesario considerar que el presente estudio presenta limitaciones relacionadas con las características de la recolección de la información, ya que gran porcentaje de sujetos contestaron de manera online y otros de manera presencial, lo que podría tener una injerencia en las respuestas de los participantes; aunque algunos estudios muestran que no habría diferencias entre estas modalidades de aplicación (Enander et al., 2012; Garrido, 2018). Por otro lado, tampoco fue posible establecer criterios externos de validación de la relación de supervisión, por lo que la validez concurrente no puede ser evaluada. Igualmente, para investigaciones futuras, se recomienda comparar con otros instrumentos que evalúen RS.

\section{Referencias}

Asociación Argentina de Estudio e Investigación en Psicodiagnóstico -ADEIP-. (2000). Pautas Internacionales para el Uso de los Tests. Versión Argentina. Argentina: ADEIP. http://www.adeip.org.ar/pautas min test.htm

Barnett, J. E., Erickson Cornish, J. A., Goodyear, R. K., \& Lichtenberg, J. W. (2007). Commentaries on the ethical and effective practice of clinical supervision. Professional Psychology: Research and Practice, 38, 268-275. https://doi. org/10.1037/07357028.38.3.268

Beinart, H. (2012). Models of supervision and the supervisory relationship. In I. Fleming \& L. Steen (Eds.), Supervision and clinical psychology: Theory, practice, and perspectives (pp. 47-62). Hove, UK: Routledge. https://psycnet.apa.org/record/2011-03314-000

Beinart, H., \& Clohessy, S. (2017). Effective Supervisory Relationships: Best Evidence and Practice. John Wiley \& Sons.

Bernard, J. M., \& Goodyear, R. K. (2019). Fundamentals of clinical supervision (6th ed.). Pearson.

Bernard, J. M., \& Goodyear, R. K. (2014). Fundamentals of clinical supervision (5th ed.). Upper Saddle River, NJ: Pearson.

Bordin, E. S. (1983). A working alliance model of supervision. Counseling Psychologist, 11, 35-42. https://doi.org/10.1177/0011000083111007

Brown, T. A. (2006). Confirmatory factor analysis for applied research. Nueva York, NY: The Guilford Press.

Cliffe, T., Beinart, H., \& Cooper, M. (2016). Development and validation of a short version of the supervisory relationship questionnaire. Clinical psychology \& psychotherapy, 23(1), 77-86. https://doi.org/10.1002/cpp.1935

Eisinga, R., Te Grotenhuis, M., \& Pelzer, B. (2013). The reliability of a two-item scale: Pearson, Cronbach, or Spearman-Brown?. International journal of public health, 58(4), 637-642. https://doi.org/10.1007/s00038-012-0416-3

Ellis, M. (2010). Bridging the science and practice of clinical supervision: Some discoveries, some misconceptions. The Clinical Supervisor, 29, 95-116. https://doi. org/10.1080/07325221003741910

Ellis, M. V., Berger, L., Hanus, A. E., Ayala, E. E., Swords, B. A., \& Siembor, M. (2013). Inadequate and harmful clinical supervision: Testing a revised framework and assessing occurrence. The Counseling Psychologist, 42(4), 434-472. https://doi. org/10.1177/0011000013508656 
Enander, J., Andersson, E., Kaldo, V., Lindefors, N., Andersson, G., \& Rück, C. (2012). Internet administration of the dimensional obsessive-compulsive scale: a psychometric evaluation. Journal of Obsessive-Compulsive and Related Disorders, 1(4), 325-330. https://doi.org/10.1016/j.jocrd.2012.07.008

Eysenck, S. B. G., Eysenck, H. J., \& Barrett, P. (1985). A revised version of the psychoticism scale. Personality and Individual Differences, 6, 21-29. https://doi. org/10.1016/0191-8869(85)90026-1

Falender, C.A., Cornish, J.A.E., Goodyear, R., Hatcher, R., Kaslow, N.J., Leventhal, G., ... Grus, C. (2004). Defining competencies in psychology supervision: A consensus statement. Journal of Clinical Psychology, 60, 771-785. https://doi.org/10.1002/ jclp.20013

Falender, C.A., \& Shafranske, E.P. (2004). Clinical supervision: A competency-based approach. Washington, DC: American Psychological Association.

Flora, D. B., \& Curran, P. J. (2004). An empirical evaluation of alternative methods of estimation for confirmatory factor analysis with ordinal data. Psychological methods, 9(4), 466. https://doi.org/10.1037/1082-989X.9.4.466

Freitas, G. J. (2002). The impact of psychotherapy supervision on client outcome: A critical examination of 2 decades of research. Psychotherapy: Theory, Research, Practice, Training, 39, 354-367. https://doi.org/10.1037/0033-3204.39.4.354

Garrido, C. I. T. (2018). Comparación entre la aplicación en formato papel y en formato online de dos instrumentos de evaluación: Cuestionario de la Forma Corporal y Cuestionario de los Tres Factores versión revisada 18. In Intervención en contextos clínicos y de la salud: Volumen II (pp. 271-278). Asociación Universitaria de Educación y Psicología -ASUNIVEP-. https://formacionasunivep.com/Vcice/ files/libro\%20intervencion $\% 20$ en $\% 20$ contextos $\% 20$ clinicos.pdf\#page $=271$

Gibson, A. S., Ellis, M. V., \& Friedlander, M. L. (2019). Toward a nuanced understanding of nondisclosure in psychotherapy supervision. Journal of Counseling Psychology, 66(1), 114. https://doi.org/10.1037/cou0000295

Gibson, J. A., Grey, I. M., \& Hastings, R. P. (2009). Supervisor support as a predictor of burnout and therapeutic self-efficacy in therapists working in ABA schools. Journal of Autism and Developmental Disorders, 39, 1024-1030. https://doi. org/10.1007/s10803-009-0709-4

Goodyear, R. K., \& Guzzardo, C. R. (2000). Psychotherapy supervision and training. In S. D. Brown \& R. W. Lent (Eds.), Handbook of counseling psychology (3rd ed., pp. 83-108). New York, NY: Wiley.

Gray, L. A., Ladany, N., Walker, J. A., \& Ancis, J. R. (2001). Psychotherapy trainees' experience of counterproductive events in supervision. Journal of Counseling Psychology, 48, 371-383-383. https://doi.org/10.1037/0022-0167.48.4.371

Hill, C., \& Knox, S. Training and supervisión in psychotherapy, in Lambert, M. J. (2013). Bergin and Garfield's handbook of psychotherapy and behavior change. John Wiley \& Sons.

Holloway, E. L. (1995). Clinical supervision: A systems approach. Thousand Oaks, CA: Sage.

Holloway, E. L. (2012). Professional competence in supervision. In J. N. Fuertes, A. Spokane, \& E. L. Holloway (Eds.), The professional competencies in counseling psychology (pp. 165-181). New York, NY: Oxford University Press.

Hu, L. T., \& Bentler, P. M. (1999). Cutoff criteria for fit indexes in covariance structure analysis: Conventional criteria versus new alternatives. Structural equation modeling: a multidisciplinary journal, 6(1), 1-55. https://doi. org/10.1080/10705519909540118 
Inman, A.G., Hutman, H., Pendse, A., Devdas, L., Luu, L., \& Ellis, M.V. (2014). Current trends concerning supervisors, supervisees, and clients in clinical supervision. In C. E. Watkins, Jr., \& D. Milne (Eds.), Wiley international handbook of clinical supervision (pp. 61-102). Oxford, UK: Wiley. https://doi.org/10.1002/9781118846360. ch4

Inman, A.G., \& Ladany, N. (2008). Research: The state of the field. In A. K. Hess, K. D. Hess, \& T. H. Hess (Eds.), Psychotherapy supervision: Theory, research, and practice (2nd ed., pp. 500-517). Hoboken, NJ: Wiley

Kennedy, E. K., Keaney, C., Shaldon, C., \& Canagaratnam, M. (2018). A relational model of supervision for applied psychology practice: professional growth through relating and reflecting. Educational Psychology in Practice, 34(3), 282-299. https:// doi.org/10.1080/02667363.2018.1456407

Kline, P. (2013). Handbook of psychological testing. Routledge.

Mehr, K. E., Ladany, N., \& Caskie, G. I. L. (2010). Trainee nondisclosure in supervision: What are they not telling you? Counselling and Psychotherapy Research, 10(2), 103-113. https://doi.org/10.1080/14733141003712301

Milne, D. L. (2017). Evidence-based CBT supervision: Principles and practice. John Wiley \& Sons.

Milne, D. L., Reiser, R. P., Cliffe, T., \& Raine, R. (2011). SAGE: Preliminary evaluation of an instrument for observing competence in CBT supervision. The Cognitive Behaviour Therapist, 4, 123-138. https://doi.org/10.1017/S1754470X11000079

Milne, D., Sheikh, A., Pattison, S., \& Wilkinson, A. (2011). Evidence-based training for clinical supervisors: A systematic review of 11 controlled studies. The Clinical Supervisor, 30(1), 53-71. https://doi.org/10.1080/07325223.2011.564955

Olk, M., \& Friedlander, M. L. (1992). Trainees' experience of role conflict and role ambiguity in supervisory relationships. Journal of Counseling Psychology, 39, 389397. https://doi.org/10.1037//00220167.39.3.389

Osborne, J. W., \& Costello, A. B. (2004). Sample size and subject to item ratio in principal components analysis. Practical assessment, research \& evaluation, 9(11), 8. https://scholarworks.umass.edu/cgi/viewcontent.cgi?article=1144\&contex$\mathrm{t}=$ pare

Palomo, M., Beinart, H., \& Cooper, M. (2010). Development and validation of the supervisory relationship questionnaire (SRQ) in UK trainee clinical psychologists. British Journal of Clinical Psychology, 49, 131-149. https://doi. org/10.1348/014466509X441033

Reiser, R.P., \& Watkins, C.E. (2014). The Wiley international handbook of clinical supervision. D. Milne (Ed.). Wiley-Blackwell.

Revelle, W. (2014). Psych: Procedures for personality and psychological research. Northwestern University, Evanston. R package version, 1(1). https://cran.r-project. org/web/packages/psych/psych.pdf

Rosseel, Y. (2012). Lavaan: An R package for structural equation modeling and more. Version 0.5-12 (BETA). Journal of statistical software, 48(2), 1-36. http://users. ugent.be/ yrosseel/lavaan/lavaanlntroduction.pdf

Scaturo, D.J., \& Watkins, C.E. (2014). Supervising integrative and eclectic psychotherapies. In C. E. Watkins, \& D. L. Milne (Eds.), The Wiley international handbook of clinical supervision (pp. 552-575). Chichester: Wiley-Blackwell.

Simon, D., Cruise, T., Huber, B., Swerdlik, M., \& Newman, D. (2014). Supervision in school psychology: The developmental/ecological/problem-solving model. Psychology in the Schools, 51(6), 636-646. https://doi.org/10.1002/pits.21772 
Stoltenberg, C.D. (2005). Enhancing professional competence through developmental approaches to supervision. American Psychologist, 60(8), 857-864. https:// doi.org/10.1037/0003-066X.60.8.85

Tangen, J.L., \& Borders, L. D. (2016). The supervisory relationship: A conceptual and psychometric review of measures. Counselor Education \& Supervision, 55(3), 159-181. https://doi.org/10.1002/ceas.12043

Watkins, C.E. (2011). Does psychotherapy supervision contribute to patient outcomes? Considering thirty years of research. The Clinical Supervisor, 30, 235256. https://doi.org/10.1080/07325223.2011.619417

Watkins, C.E. (2012). Psychotherapy supervision in the new millennium: Competency-based, evidence-based, particularized, and energized. Journal of Contemporary Psychotherapy, 42, 193-203. https://doi.org/10.1007/s10879-011-9202-4

Watkins, C.E., Jr. (2018, May 24). The Generic Model of Psychotherapy Supervision: An Analogized Research-Informing Meta-Theory. Journal of Psychotherapy Integration. Advance online publication. https://doi.org/10.1037/int0000114

Watkins, C.E. (2014). The supervisory alliance: A half century of theory, practice and research in critical perspective. American Journal of Psychotherapy, 68, 1-37. https://doi.org/10.1176/appi.psychotherapy.2014.68.1.19

Wheeler, S., \& Richards, K. (2007). The impact of clinical supervision on counselors and therapists, their practice and their clients: A systematic review of the literature. Counselling \& Psychotherapy Research, 7, 54-65. https://doi. org/10.1080/14733140601185274 